\title{
Fekete-Szegö Problem for Certain New Family of Bi-Univalent Functions
}

\author{
Abbas Kareem Wanas ${ }^{1}$ and Haeder Younis Althoby ${ }^{2}$ \\ ${ }^{1}$ Department of Mathematics, College of Science, University of Al-Qadisiyah, Iraq \\ e-mail: abbas.kareem.w@qu.edu.iq \\ ${ }^{2}$ Department of Mathematics, College of Science, University of Al-Qadisiyah, Iraq \\ e-mail: hayder.younis@qu.edu.iq
}

\begin{abstract}
In current effort, by making use of the principle of subordination, we introduce and study a new family of holomorphic and bi-univalent functions which are defined in open unit disk and solve Fekete-Szegö problem for functions which belong to this family.
\end{abstract}

\section{Introduction and Preliminaries}

Indicate by $\mathcal{A}$ the family of functions $\mathfrak{U}$ that are holomorphic in the unit disk $\mathfrak{D}=$ $\{t \in \mathbb{C}:|t|<1\}$ which have the shape:

$$
\mathfrak{U}(t)=t+\sum_{n=2}^{\infty} a_{n} t^{n}
$$

Assume that $\mathcal{S}$ stand for the subfamily of $\mathcal{A}$ containing of the shape (1.1) that are univalent in $\mathfrak{D}$.

The Fekete-Szegö functional $\left|a_{3}-\mu a_{2}^{2}\right|$ for $\mathfrak{U} \in \mathcal{S}$ is well known for its rich history in the field of "Geometric Function Theory". Its origin was in the disproof by Fekete and Szegö [12] conjecture of Littlewood and Paley that the coefficients of odd univalent functions are bounded by unity. The functional has since received great attention, particularly in many sub families of the family of univalent functions. Nowadays, it

Received: December 3, 2021; Accepted: January 4, 2022

2010 Mathematics Subject Classification: 30C45, 30C50.

Keywords and phrases: holomorphic functions, bi-univalent functions, Fekete-Szegö problem, subordination, coefficient bounds. 
seems that this topic had become an interest among the researchers (see, for example, $[5$, 19]).

For two functions $\mathfrak{F}$ and $\mathcal{Y}$, holomorphic in the unit disk $\mathfrak{D}$, we name that the function $\mathfrak{F}(t)$ is subordinate to $\mathcal{Y}(t)$ in $\mathfrak{D}$, and write

$$
\mathfrak{F}(t) \prec \mathcal{Y}(t)(t \in \mathfrak{D}),
$$

if there is a Schwarz function $\mathfrak{S}(t)$, holomorphic in $\mathfrak{D}$, with

$$
\mathfrak{S}(0)=0 \text { and }|\Im(t)|<1 \quad(t \in \mathfrak{D}) \text {, }
$$

such that

$$
\mathfrak{F}(t)=\mathcal{Y}(\mathfrak{S}(t))(t \in \mathfrak{D})
$$

In special, if the function $\mathcal{Y}$ is univalent in $\mathfrak{D}$, then

$$
\mathfrak{F}(0)=\mathcal{Y}(0) \text { and } \mathfrak{F}(\mathfrak{D}) \subset \mathcal{Y}(\mathfrak{D})
$$

It is well known (see [11]) that each function $\mathfrak{U} \in \mathcal{S}$ has an inverse $\mathfrak{U}^{-1}$, defined by

$$
\mathfrak{U}^{-1}(\mathfrak{U}(t))=t, \quad(t \in \mathfrak{D})
$$

and

$$
\mathfrak{U}\left(\mathfrak{U}^{-1}(\mathfrak{M})\right)=\mathfrak{M}, \quad\left(|\mathfrak{M}|<r_{0}(\mathfrak{U}), r_{0}(\mathfrak{U}) \geq \frac{1}{4}\right)
$$

where

$$
\begin{aligned}
\mathfrak{B}(\mathfrak{B}) & =\mathfrak{U}^{-1}(\mathfrak{B}) \\
& =\mathfrak{B}-a_{2} \mathfrak{B}^{2}+\left(2 a_{2}^{2}-a_{3}\right) \mathfrak{B}^{3}-\left(5 a_{2}^{3}-5 a_{2} a_{3}+a_{4}\right) \mathfrak{B}^{4}+\cdots
\end{aligned}
$$

A function $\mathfrak{U} \in \mathcal{A}$ is named bi-univalent in $\mathfrak{D}$ if both $\mathfrak{U}$ and $\mathfrak{U}^{-1}$ are univalent in $\mathfrak{D}$. Let $\Sigma$ denotes the family of all bi-univalent functions in $\mathfrak{D}$ that satisfy (1.1). In fact, Srivastava et al. [22] refreshed the study of holomorphic bi-univalent functions in recent years, it was followed by such works (see $[1,2,4,6,8,9,10,13,14,16,17,18,20,21,23,24$, 26,27]).

We shall need the next lemma to establish the desired bounds in our investigation.

Lemma 1.1 [16]. If $\mathcal{T}(t)=1+\mathcal{T}_{1} t+\mathcal{T}_{2} t^{2}+\mathcal{T}_{3} t^{3}+\cdots$ is an holomorphic function in $\mathfrak{D}$ with positive real part, then

$$
\left|\mathcal{T}_{n}\right| \leq 2 \quad(n \in \mathbb{N}=\{1,2,3, \ldots\})
$$


and

$$
\left|\mathcal{T}_{2}-\frac{\mathcal{T}_{1}^{2}}{2}\right| \leq 2-\frac{\left|\mathcal{T}_{1}\right|^{2}}{2}
$$

\section{Main Results}

In the sequel, symbolized by $\vartheta$ the holomorphic function with positive real part in $\mathfrak{D}$, satisfying $\vartheta(0)=1$ and $\vartheta^{\prime}(0)>0$. Also, assume that $\vartheta(\mathcal{D})$ be starlike with respect to 1 and symmetric with respect to the real axis. Such a function $\vartheta$ has the Taylor series expansion of the shape:

$$
\vartheta(t)=1+\xi_{1} t+\xi_{2} t^{2}+\xi_{3} t^{3}+\cdots, \quad \xi_{1}>0 .
$$

Definition 2.1. A function $\mathfrak{U} \in \Sigma$ given by (1.1) is named in the family $F_{\Sigma}(\gamma, \eta, \tau ; \vartheta)$, $(0 \leq \gamma \leq 1,0 \leq \eta \leq 1,0 \leq \tau \leq 1)$ if it fulfills the next subordinations:

$$
\left(\frac{t \mathfrak{U}^{\prime}(t)}{\mathfrak{U}(t)}\right)^{\gamma}\left[(1-\tau) \frac{t \mathfrak{U}^{\prime}(t)}{\mathfrak{U}(t)}+\tau\left(1+\frac{t \mathfrak{U}^{\prime \prime}(t)}{\mathfrak{U}^{\prime}(t)}\right)\right]^{\eta} \prec \vartheta(t)
$$

and

$$
\left(\frac{\mathfrak{W B}^{\prime}(\mathfrak{B})}{\mathfrak{V}(\mathfrak{M})}\right)^{\gamma}\left[(1-\tau) \frac{\mathfrak{W} \mathfrak{B}^{\prime}(\mathfrak{B})}{\mathfrak{B}(\mathfrak{B})}+\tau\left(1+\frac{\mathfrak{B B}^{\prime \prime}(\mathfrak{B})}{\mathfrak{V}^{\prime}(\mathfrak{B})}\right)\right]^{\eta} \prec \vartheta(\mathfrak{B}),
$$

where the function $\mathfrak{B}=\mathfrak{U}^{-1}$ is given by (1.2).

Remark 2.1. It should be remarked that the family $F_{\Sigma}(\gamma, \eta, \tau ; \vartheta)$ is a generalization of well-known families considered earlier. These families are:

(1) For $\gamma=0$ and $\eta=1$, the family $F_{\Sigma}(\gamma, \eta, \tau ; \vartheta)$ reduce to the family $\mathcal{M}_{\Sigma}(\eta ; \vartheta)$ which was introduced recently by Ali et al. [3].

(2) For $\gamma=0, \eta=1$ and $\vartheta(t)=\left(\frac{1+t}{1-t}\right)^{\alpha}, 0<\alpha \leq 1$, the family $F_{\Sigma}(\gamma, \eta, \tau ; \vartheta)$ reduce to the family $M_{\Sigma}(\alpha, \tau)$ which was investigated by Liu and Wang [15].

(3) For $\gamma=0, \eta=1$ and $\vartheta(t)=\frac{1+(1-2 \beta) t}{1-t}, 0 \leq \beta<1$, the family $F_{\Sigma}(\gamma, \eta, \tau ; \vartheta)$ reduce to the family $B_{\Sigma}(\beta, \tau)$ which was studied by Liu and Wang [15].

(4) For $\gamma=\tau=0, \eta=1$ and $\vartheta(t)=\left(\frac{1+t}{1-t}\right)^{\alpha}, 0<\alpha \leq 1$, the family $F_{\Sigma}(\gamma, \eta, \tau ; \vartheta)$ reduce to the family $S_{\Sigma}^{*}(\alpha)$ which was introduced by Brannan and Taha [7]. 
(5) For $\gamma=\tau=0, \eta=1$ and $\vartheta(t)=\frac{1+(1-2 \beta) t}{1-t}, 0 \leq \beta<1$, the family $F_{\Sigma}(\gamma, \eta, \tau ; \vartheta)$ reduce to the family $S_{\Sigma}^{*}(\beta)$ which was studied by Brannan and Taha [7].

(6) For $\tau=1$ and $\eta=1-\gamma$, the family $F_{\Sigma}(\gamma, \eta, \tau ; \vartheta)$ reduce to the family $\mathcal{L}_{\Sigma}(\gamma ; \vartheta)$ which was investigated by Ali et al. [3].

(7) For $\tau=1, \gamma=1-\eta$ and $\vartheta(t)=\frac{1+t}{1-t}$ the family $F_{\Sigma}(\gamma, \eta, \tau ; \vartheta)$ reduce to the family $\mathcal{M}^{\eta}$ which was considered recently by Thomas [25].

Theorem 2.1. Let $\mathfrak{U} \in F_{\Sigma}(\gamma, \eta, \tau ; \vartheta), \mu \in \mathbb{R}$. Then

$$
\leq\left\{\begin{array}{c}
\left|a_{3}-\mu a_{2}^{2}\right| \\
\times\left|\gamma(\gamma+1)+\eta(\tau+1)(2(\gamma+1)+(\eta-1)(\tau+1))+2(\gamma+\eta(\tau+1))^{2} \frac{\xi_{1}-\xi_{2}}{\xi_{1}^{2}}\right| \\
\frac{|\mu-1| \xi_{1}^{3}}{2(\gamma+\eta(2 \tau+1))}, \quad \mathfrak{U} \text { or }|\mu-1| \leq \frac{1}{4(\gamma+\eta(2 \tau+1))} \times \\
\qquad \begin{array}{c}
\mathfrak{U} \text { or }|\mu-1| \geq \frac{1}{4(\gamma+\eta(2 \tau+1))} \times \\
\times\left|\gamma(\gamma+1)+\eta(\tau+1)(2(\gamma+1)+(\eta-1)(\tau+1))+2(\gamma+\eta(\tau+1))^{2} \frac{\xi_{1}-\xi_{2}}{\xi_{1}^{2}}\right| .
\end{array}
\end{array}\right.
$$

Proof. Assume that $\mathfrak{U} \in F_{\Sigma}(\gamma, \eta, \tau ; \vartheta)$. Then there are two holomorphic functions $u, v: \mathfrak{D} \rightarrow \mathfrak{D}$ with $u(0)=v(0)=0,|u(t)|<1,|v(\mathfrak{B})|<1$ and $t, \mathfrak{B} \in \mathfrak{D}$ such that

$$
\left(\frac{t \mathfrak{U}^{\prime}(t)}{\mathfrak{U}(t)}\right)^{\gamma}\left[(1-\tau) \frac{t \mathfrak{U}^{\prime}(t)}{\mathfrak{U}(t)}+\tau\left(1+\frac{t \mathfrak{U}^{\prime \prime}(t)}{\mathfrak{U}^{\prime}(t)}\right)\right]^{\eta}=\vartheta(u(t))
$$

and

$$
\left(\frac{\mathfrak{W}^{\prime}(\mathfrak{B})}{\mathfrak{B}(\mathfrak{B})}\right)^{\gamma}\left[(1-\tau) \frac{\mathfrak{W B}^{\prime}(\mathfrak{M})}{\mathfrak{B}(\mathfrak{B})}+\tau\left(1+\frac{\mathfrak{B V}^{\prime \prime}(\mathfrak{B})}{\mathfrak{B}^{\prime}(\mathfrak{B})}\right)\right]^{\eta}=\vartheta(v(\mathfrak{B})),
$$

where $\mathfrak{B}=\mathfrak{U}^{-1}$.

We define the functions $\mathcal{T}$ and $\mathfrak{I}$ by

$$
\mathcal{T}(t)=\frac{1+u(t)}{1-u(t)}=1+\mathcal{T}_{1} t+\mathcal{T}_{2} t^{2}+\cdots
$$


and

$$
\mathfrak{I}(\mathfrak{B})=\frac{1+v(\mathfrak{B})}{1-v(\mathfrak{B})}=1+\mathfrak{I}_{1} \mathfrak{W}+\mathfrak{I}_{2} \mathfrak{W}^{2}+\cdots
$$

It is clear that $\mathcal{T}$ and $\mathfrak{I}$ are holomorphic in $\mathfrak{D}$ and $\mathcal{T}(0)=\mathfrak{I}(0)=1$. Since $u, v: \mathfrak{D} \rightarrow \mathfrak{D}$, then the functions $\mathcal{T}$ and $\mathfrak{I}$ have positive real part in $\mathfrak{D}$.

It follows from (2.4) and (2.5) that

$$
u(t)=\frac{\mathcal{T}(t)-1}{\mathcal{T}(t)+1}=\frac{1}{2} \mathcal{T}_{1} t+\frac{1}{2}\left(\mathcal{T}_{2}-\frac{1}{2} \mathcal{T}_{1}^{2}\right) t^{2}+\cdots
$$

and

$$
v(\mathfrak{B})=\frac{\mathfrak{T}(\mathfrak{B})-1}{\mathfrak{I}(\mathfrak{B})+1}=\frac{1}{2} \mathfrak{I}_{1} \mathfrak{W}+\frac{1}{2}\left(\mathfrak{I}_{2}-\frac{1}{2} \mathfrak{T}_{1}^{2}\right) \mathfrak{M}^{2}+\cdots .
$$

Using (2.6) and (2.7) together with (2.1), we easily obtain

$$
\vartheta(u(t))=1+\frac{1}{2} \xi_{1} \mathcal{T}_{1} t+\frac{1}{2}\left(\frac{1}{2} \xi_{2} \mathcal{T}_{1}^{2}+\xi_{1}\left(\mathcal{T}_{2}-\frac{1}{2} \mathcal{T}_{1}^{2}\right)\right) t^{2}+\cdots
$$

and

$$
\vartheta(v(\mathfrak{W}))=1+\frac{1}{2} \xi_{1} \mathfrak{I}_{1} \mathfrak{W}+\frac{1}{2}\left(\frac{1}{2} \xi_{2} \mathfrak{I}_{1}^{2}+\xi_{1}\left(\mathfrak{I}_{2}-\frac{1}{2} \mathfrak{I}_{1}^{2}\right)\right) \mathfrak{W}^{2}+\cdots
$$

Since

$$
\begin{aligned}
& \left(\frac{t \mathfrak{U}^{\prime}(t)}{\mathfrak{U}(t)}\right)^{\gamma}\left[(1-\tau) \frac{t \mathfrak{U}^{\prime}(t)}{\mathfrak{U}(t)}+\tau\left(1+\frac{t \mathfrak{U}^{\prime \prime}(t)}{\mathfrak{U}^{\prime}(t)}\right)\right]^{\eta}=1+(\gamma+\eta(\tau+1)) a_{2} t \\
& +\left[2(\gamma+\eta(2 \tau+1)) a_{3}\right. \\
& \left.+\frac{1}{2}[\gamma(\gamma-1)+\eta(\tau+1)(2 \gamma+(\eta-1)(\tau+1))-2(\gamma+\eta(3 \tau+1))] a_{2}^{2}\right] t^{2}+\cdots
\end{aligned}
$$

and

$$
\begin{aligned}
& \left(\frac{\mathfrak{B S}^{\prime}(\mathfrak{B})}{\mathfrak{B}(\mathfrak{B})}\right)^{\gamma}\left[(1-\tau) \frac{\mathfrak{B V}^{\prime}(\mathfrak{B})}{\mathfrak{B}(\mathfrak{B})}+\tau\left(1+\frac{\mathfrak{B O}^{\prime \prime}(\mathfrak{B})}{\mathfrak{B}^{\prime}(\mathfrak{B})}\right)\right]^{\eta}=1-(\gamma+\eta(\tau+1)) a_{2} \mathfrak{M} \\
& +\left[\frac{1}{2}[\gamma(\gamma-1)+\eta(\tau+1)(2 \gamma+(\eta-1)(\tau+1))+2(3 \gamma+\eta(5 \tau+3))] a_{2}^{2}\right.
\end{aligned}
$$


$\left.-2(\gamma+\eta(2 \tau+1)) a_{3}\right] \mathfrak{M}^{2}+\cdots$,

then (2.2), (2.3), (2.8) and (2.9) yields

$$
\begin{gathered}
(\gamma+\eta(\tau+1)) a_{2}=\frac{1}{2} \xi_{1} \mathcal{T}_{1} \\
2(\gamma+\eta(2 \tau+1)) a_{3} \\
+\frac{1}{2}[\gamma(\gamma-1)+\eta(\tau+1)(2 \gamma+(\eta-1)(\tau+1))-2(\gamma+\eta(3 \tau+1))] a_{2}^{2} \\
=\frac{1}{2}\left(\frac{1}{2} \xi_{2} \mathcal{T}_{1}^{2}+\xi_{1}\left(\mathcal{T}_{2}-\frac{1}{2} \mathcal{T}_{1}^{2}\right)\right) \\
-(\gamma+\eta(\tau+1)) a_{2}=\frac{1}{2} \xi_{1} \mathfrak{I}_{1}
\end{gathered}
$$

and

$$
\begin{array}{r}
\frac{1}{2}[\gamma(\gamma-1)+\eta(\tau+1)(2 \gamma+(\eta-1)(\tau+1))+2(3 \gamma+\eta(5 \tau+3))] a_{2}^{2} \\
-2(\gamma+\eta(2 \tau+1)) a_{3}=\frac{1}{2}\left(\frac{1}{2} \xi_{2} \mathfrak{I}_{1}^{2}+\xi_{1}\left(\mathfrak{I}_{2}-\frac{1}{2} \mathfrak{I}_{1}^{2}\right)\right) .
\end{array}
$$

In view of (2.10) and (2.12) that

$$
\mathcal{T}_{1}=-\mathfrak{I}_{1}
$$

and

$$
8(\gamma+\eta(\tau+1))^{2} a_{2}^{2}=\xi_{1}^{2}\left(\mathcal{T}_{2}^{2}+\mathfrak{T}_{2}^{2}\right) .
$$

By subtracting (2.11) from (2.13) and using (2.14), we find that

$$
a_{3}=a_{2}^{2}+\frac{\xi_{1}\left(\mathcal{T}_{2}-\mathfrak{I}_{2}\right)}{8(\gamma+\eta(2 \tau+1))} .
$$

If we add (2.11) to (2.13), we deduce that

$$
\begin{aligned}
& {[\gamma(\gamma+1)+\eta(\tau+1)(2(\gamma+1)+(\eta-1)(\tau+1))] a_{2}^{2} } \\
= & \frac{1}{2}\left[\xi_{1}\left(\mathcal{T}_{2}+\mathfrak{I}_{2}\right)-\frac{1}{2}\left(\xi_{1}-\xi_{2}\right)\left(\mathcal{T}_{1}^{2}+\mathfrak{I}_{1}^{2}\right)\right] .
\end{aligned}
$$

By substitute the value of $\mathcal{T}_{1}^{2}+\mathfrak{I}_{1}^{2}$ from (2.15) in the right hand side of (2.17), we get 


$$
a_{2}^{2}=\frac{\xi_{1}^{3}\left(\mathcal{T}_{2}+\mathfrak{I}_{2}\right)}{2[\gamma(\gamma+1)+\eta(\tau+1)(2(\gamma+1)+(\eta-1)(\tau+1))] \xi_{1}^{2}} .
$$

From (2.16) and (2.18), we obtain

$$
a_{3}-\mu a_{2}^{2}=\xi_{1}\left[\left(\psi(\mu)+\frac{1}{8(\gamma+\eta(2 \tau+1))}\right) \mathcal{T}_{2}+\left(\psi(\mu)-\frac{1}{8(\gamma+\eta(2 \tau+1))}\right) \mathfrak{I}_{2}\right],
$$

where

$$
\psi(\mu)=\frac{(1-\mu) \xi_{1}^{2}}{2[\gamma(\gamma+1)+\eta(\tau+1)(2(\gamma+1)+(\eta-1)(\tau+1))] \xi_{1}^{2}} .
$$

According to Lemma 1.1 and (2.1), we conclude that

$$
\left|a_{3}-\mu a_{2}^{2}\right| \leq\left\{\begin{array}{lc}
\frac{\xi_{1}}{2(\gamma+\eta(2 \tau+1))}, & 0 \leq|\psi(\mu)| \leq \frac{1}{8(\gamma+\eta(2 \tau+1))} \\
4 \xi_{1}|\psi(\mu)|, & |\psi(\mu)| \geq \frac{1}{8(\gamma+\eta(2 \tau+1))},
\end{array}\right.
$$

After some computations, we deduce that

$$
\begin{aligned}
& \left|a_{3}-\mu a_{2}^{2}\right| \\
& \leq\left\{\begin{array}{c}
\frac{\xi_{1}}{2(\gamma+\eta(2 \tau+1))}, \quad \text { for }|\mu-1| \leq \frac{1}{4(\gamma+\eta(2 \tau+1))} \times \\
\times\left|\gamma(\gamma+1)+\eta(\tau+1)(2(\gamma+1)+(\eta-1)(\tau+1))+2(\gamma+\eta(\tau+1))^{2} \frac{\xi_{1}-\xi_{2}}{\xi_{1}^{2}}\right| \\
\quad \text { for }|\mu-1| \geq \frac{1}{4(\gamma+\eta(2 \tau+1))} \times \\
\times\left|\gamma(\gamma+1)+\eta(\tau+1)(2(\gamma+1)+(\eta-1)(\tau+1))+2(\gamma+\eta(\tau+1))^{2} \frac{\xi_{1}-\xi_{2}}{\xi_{1}^{2}}\right| .
\end{array}\right.
\end{aligned}
$$

Taking $\mu=1$ in Theorem 2.1, we conclude the next outcome:

Corollary 2.1. If $\mathfrak{U} \in F_{\Sigma}(\gamma, \eta, \tau ; \vartheta)$, then 


$$
\left|a_{3}-a_{2}^{2}\right| \leq \frac{\xi_{1}}{2(\gamma+\eta(2 \tau+1))} .
$$

Taking $\mu=0$ in Theorem 2.1, we conclude the next outcome:

Corollary 2.2. If $\mathfrak{U} \in F_{\Sigma}(\gamma, \eta, \tau ; \vartheta)$, then

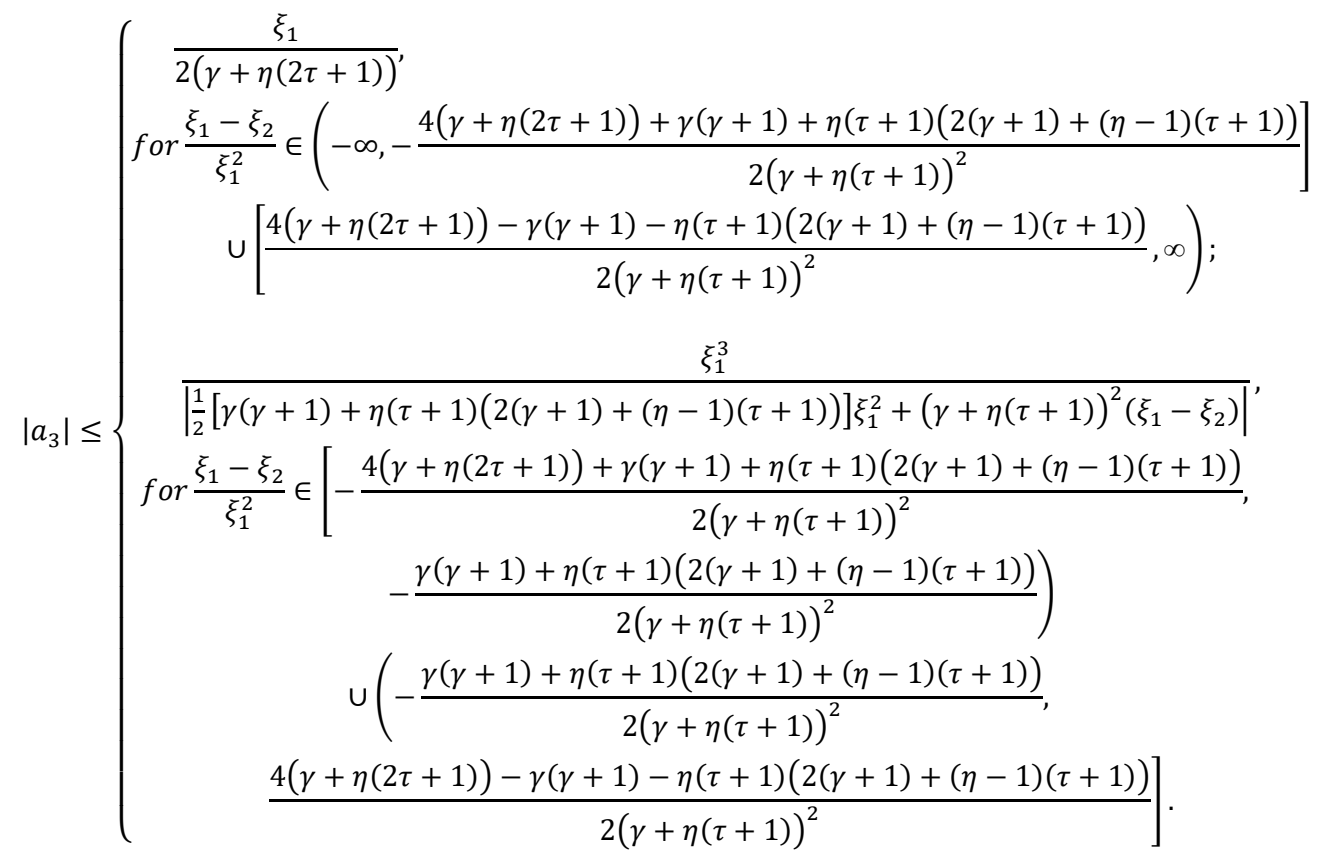

\section{References}

[1] C. Abirami, N. Magesh and J. Yamini, Initial bounds for certain classes of bi-univalent functions defined by Horadam polynomials, Abstr. Appl. Anal. 2020, Art. ID 7391058, 8 pp. https://doi.org/10.1155/2020/7391058

[2] A. G. Al-Amoush, Certain subclasses of bi-univalent functions involving the Poisson distribution associated with Horadam polynomials, Malaya J. Mat. 7 (2019), 618-624. https://doi.org/10.26637/MJM0704/0003

[3] R. M. Ali, S. K. Lee, V. Ravichandran and S. Supramaniam, Coefficient estimates for biunivalent Ma-Minda starlike and convex functions, Appl. Math. Lett. 25 (2012), 344-351. https://doi.org/10.1016/j.aml.2011.09.012

[4] A. Amourah, B. A. Frasin, G. Murugusundaramoorthy and T. Al-Hawary, Bi-Bazilevic functions of order $\vartheta+i \delta$ associated with $(p, q)$-Lucas polynomials, AIMS Mathematics 6(5) (2021), 4296-4305. https://doi.org/10.3934/math.2021254 
[5] S. Altinkaya and S. Yalçin, Fekete-Szegö inequalities for certain classes of bi-univalent functions, International Scholarly Research Notices 2014, Art. ID 327962, 6 pp. https://doi.org/10.1155/2014/327962

[6] S. Altinkaya and S. Yalçin, Coefficient estimates for two new subclasses of bi-univalent functions with respect to symmetric points, J. Funct. Spaces 2015, Art. ID 145242, 5 pp. https://doi.org/10.1155/2015/145242

[7] D. A. Brannan and T. S. Taha, On some classes of bi-univalent functions, Studia Univ. Babeş-Bolyai Math. 31(2) (1986), 70-77.

[8] S. Bulut, Faber polynomial coefficient estimates for a subclass of analytic bi-univalent functions, Filomat 30 (2016), 1567-1575. https://doi.org/10.2298/FIL1606567B

[9] M. Caglar, E. Deniz and H. M. Srivastava, Second Hankel determinant for certain subclasses of bi-univalent functions, Turkish J. Math. 41 (2017), 694-706. https://doi.org/10.3906/mat-1602-25

[10] M. Caglar, H. Orhan and N. Yagmur, Coefficient bounds for new subclasses of biunivalent functions, Filomat 27 (2013), 1165-1171. https://doi.org/10.2298/FIL1307165C

[11] P. L. Duren, Univalent Functions, Grundlehren der Mathematischen Wissenschaften, Band 259, Springer Verlag, New York, Berlin, Heidelberg and Tokyo, 1983.

[12] M. Fekete and G. Szegö, Eine Bemerkung Über Ungerade Schlichte Funktionen, J. London Math. Soc. 8 (1933), 85-89. https://doi.org/10.1112/jlms/s1-8.2.85

[13] B. A. Frasin and M. K. Aouf, New subclasses of bi-univalent functions, Appl. Math. Lett. 24 (2011), 1569-1573. https://doi.org/10.1016/j.aml.2011.03.048

[14] H. O. Guney, G. Murugusundaramoorthy and J. Sokol, Subclasses of bi-univalent functions related to shell-like curves connected with Fibonacci numbers, Acta Univ. Sapientiae Math. 10 (2018), 70-84. https://doi.org/10.2478/ausm-2018-0006

[15] X. F. Li and A. P. Wang, Two new subclasses of bi-univalent functions, Int. Math. Forum 7(2) (2012), 1495-1504.

[16] N. Magesh and V. Prameela, Coefficient estimate problems for certain subclasses of analytic and bi-univalent functions, Afr. Mat. 26(3) (2013), 465-470. https://doi.org/10.1007/s13370-013-0220-0

[17] E. P. Mazi and T. O. Opoola, On some subclasses of bi-univalent functions associating pseudo-starlike functions with Sakaguchi type functions, General Mathematics 25 (2017), 85-95.

[18] H. Orhan, N. Magesh and V. K. Balaji, Initial coefficient bounds for a general class of biunivalent functions, Filomat 29 (2015), 1259-1267. https://doi.org/10.2298/FIL1506259O 
[19] Ch. Pommerenke, Univalent Functions, Vandenhoeck and Rupercht, Göttingen, 1975.

[20] H. M. Srivastava, S. S. Eker and R. M. Ali, Coefficient bounds for a certain class of analytic and bi-univalent functions, Filomat 29 (2015), 1839-1845. https://doi.org/10.2298/FIL1508839S

[21] H. M. Srivastava, S. S. Eker, S. G. Hamidi and J. M. Jahangiri, Faber polynomial coefficient estimates for bi-univalent functions defined by the Tremblay fractional derivative operator, Bull. Iranian Math. Soc. 44(1) (2018), 149-157. https://doi.org/10.1007/s41980-018-0011-3

[22] H. M. Srivastava, A. K. Mishra and P. Gochhayat, Certain subclasses of analytic and biunivalent functions, Appl. Math. Lett. 23 (2010), 1188-1192. https://doi.org/10.1016/j.aml.2010.05.009

[23] H. M. Srivastava, A. Motamednezhad and E. A. Adegani, Faber polynomial coefficient estimates for bi-univalent functions defined by using differential subordination and a certain fractional derivative operator, Mathematics 8 (2020), Art. ID 172. https://doi.org/10.3390/math8020172

[24] H. M. Srivastava, A. K. Wanas and R. Srivastava, Applications of the $q$-Srivastava-Attiya operator involving a certain family of bi-univalent functions associated with the Horadam polynomials, Symmetry 13 (2021), Art. ID 1230. https://doi.org/10.3390/sym13071230

[25] D. K. Thomas, On the coefficients of Gamma-starlike functions, J. Korean Math. Soc. 55(1) (2018), 175-184.

[26] A. K. Wanas, New families of bi-univalent functions governed by Gegenbauer polynomials, Earthline J. Math. Sci. 7(2) (2021), 403-427.

https://doi.org/10.34198/ejms.7221.403427

[27] P. Zaprawa, On the Fekete-Szegö problem for classes of bi-univalent functions, Bull. Belg. Math. Soc. Simon Stevin 21 (2014), 169-178.

https://doi.org/10.36045/bbms/1394544302

This is an open access article distributed under the terms of the Creative Commons Attribution License (http://creativecommons.org/licenses/by/4.0/), which permits unrestricted, use, distribution and reproduction in any medium, or format for any purpose, even commercially provided the work is properly cited. 